\title{
Vibrational spectrum of magnesium hydride
}

\author{
J. R. Santisteban, * G. J. Cuello, ${ }^{*}$ J. Dawidowski, $^{*}$ A. Fainstein, ${ }^{*}$ and H. A. Peretti \\ Centro Atómico Bariloche (CNEA), Bustillo 9500, 8400 Bariloche (RN), Argentina
}

\author{
A. Ivanov \\ Institut Laue Langevin, Boîte Postale 156, F-38042 Grenoble Cedex 9, France
}

\author{
F. J. Bermejo
}

Instituto de Estructura de la Materia, Consejo Superior de Investigaciones Científicas Serrano 123, Madrid E-28006, Spain

(Received 20 April 1999; revised manuscript received 22 February 2000)

\begin{abstract}
The vibrational spectrum of hydrogen atoms in magnesium hydride has been studied by means of inelasticneutron and Raman-scattering experiments between 12 and $295 \mathrm{~K}$. After careful evaluation of multiple scattering and multiphonon contributions, we provide a vibrational frequency spectrum up to $180 \mathrm{meV}$ and the mean-square displacement of hydrogen atoms. The inelastic light scattering spectra of $\mathrm{MgH}_{2}$ powders show three Raman allowed modes $\left(A_{1 g}, B_{1 g}\right.$, and $\left.E_{g}\right)$ and some smaller features that can be related with secondorder and disorder-induced processes.
\end{abstract}

Hydrogen forms almost ionic hydrides by reaction with all of the alkali and alkaline-earth metals. In these materials, evidence seems consistent with hydrogen being present in the form of anions or modified anions. On the other hand, it reacts exothermically to form metallic, semimetallic, and semiconducting hydrides with the transition metals in groups III, IV, and V as well as with the rare-earth metals and the actinide metals. The transition metals in groups VI, VII, and VIII react endothermically and generally do not form stable hydrides, with the notable exception of palladium.

In groups-III-VIII metal hydrides, the proton can be seen as an impurity, and it is possible to imagine a continuous transformation between metal and hydride lattices, produced by distortions in the primitive metal lattice due to the filling of the interstitial sites. The dynamics of the proton within these metal hydrides has been the subject of great interest in the past decades, motivated by the high mobility of hydrogen, the existence of almost dispersionless modes of vibration, and the relation between superconductivity and optic vibrations. On the other hand, the structure and dynamics of the proton is quite different in the hydrides of alkali and alkaline-earth metals (e.g., $\mathrm{MgH}_{2}$ ), where long-range interactions exist between hydrogen atoms. ${ }^{1}$ There is a lack of experimental and theoretical studies concerning the dynamics of hydrogen in these almost ionic hydrides. In contrast to this situation, the large coherent cross section of fluorine atoms makes possible the determination of the dispersion curves, and much work has been carried out on alkaline and alkaline-earth fluorides compounds $\left(\mathrm{MgF}_{2}, \mathrm{BaF}_{2}, \mathrm{NaF}\right.$, etc. $\left.^{2}\right)$, which are usually isomorphous with the corresponding hydrides. In addition, extensive Raman results have been reported for these latter compounds, providing accurate values of the frequencies and symmetries of the Raman-allowed vibrations. ${ }^{3,4}$ Although these results provide starting points to predict the actual dynamics of the hydrides, a more detailed study will require direct experimental data. An early work ${ }^{5}$ reports the neutron inelastic spectra of $\mathrm{CaH}_{2}, \mathrm{SrH}_{2}$, and
$\mathrm{BaH}_{2}$, although the poor resolution of the experiments did not allow a precise definition of the hydrogen vibrations.

In the present work, we study the vibrational density of states of magnesium hydride by means of inelastic-neutron and Raman-scattering experiments. Because of the large incoherent cross section of hydrogen, this contribution dominates the neutron-scattering spectra, allowing the extraction of the phonon density of states (DOS) from the experimental data. On the other hand, the Raman spectra enable an additional check and provide valuable symmetry information to identify the phonon bands in the vibrational DOS.

Magnesium hydride has the rutile structure with $\mathrm{H}$ atoms forming a slightly distorted hcp lattice, while the $\mathrm{Mg}$ atoms occupy half of the available octahedral sites. ${ }^{6}$ The samples used were powders produced from metal turnings and gaseous hydrogen in a fixed volume vessel at $500{ }^{\circ} \mathrm{C}$. It follows from the analysis of detailed micrographies that $\mathrm{MgH}_{2}$ hydride forms 30-60 $\mu \mathrm{m}$ layers surrounding unreacted $\alpha$ (metal) phase at the cores of the turnings. The overall composition, $\mathrm{MgH}_{0.5}$, was obtained from the change in the $\mathrm{H}$ pressure within the reactor, and was subsequently confirmed by a neutron-scattering technique described elsewhere. ${ }^{7}$ The lattice parameters at room temperature of the rutile structure, $a=4.525 \AA$ and $c=3.025 \AA$, were defined by x-ray diffraction experiments, and these values are in good agreement with the data found in the literature.

The inelastic-neutron scattering (INS) experiments were performed at the high-flux reactor located at the Institut Laue-Langevin (Grenoble, France) using the triple-axis spectrometer IN1, and the analysis of energy was made using a liquid-nitrogen-cooled beryllium filter to select neutrons with final energies below $5.2 \mathrm{meV}$. Monochromatic neutrons with energies between 20 and $170 \mathrm{meV}$ were employed and the detectors were positioned at $\theta=90^{\circ}$. The inelastic spectra were taken at 295, 200, 150, 100, 50, and $12 \mathrm{~K}$. An extra measurement at $12 \mathrm{~K}$ with less flux but improved resolution was performed, thus verifying that the instrument resolution is not important to define the relevant features of the spectra. 


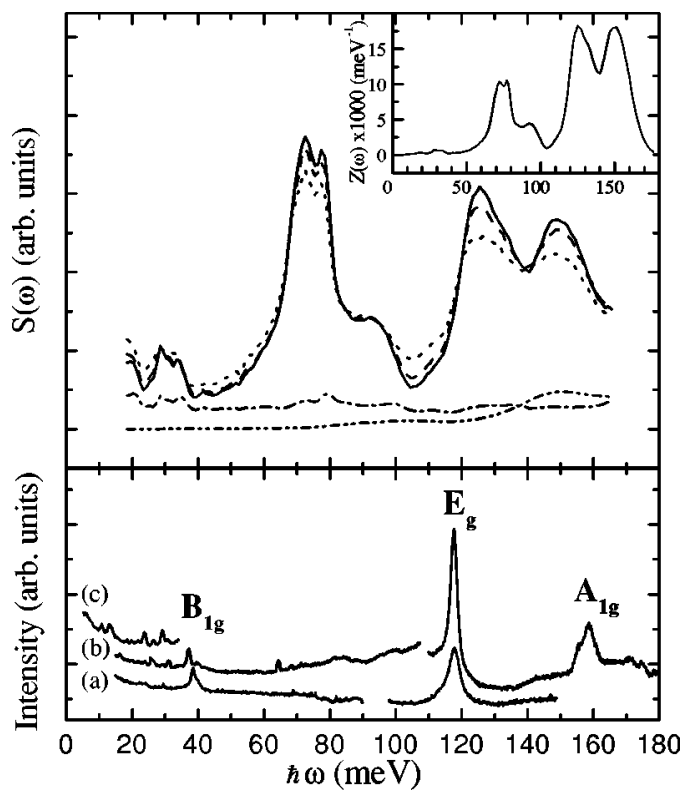

FIG. 1. Top: Neutron inelastic spectra of $\mathrm{MgH}_{2}$ for $12 \mathrm{~K}$ (solid line), $150 \mathrm{~K}$ (dashed line), and $295 \mathrm{~K}$ (dotted line), together with the multiple scattering (dashed-dotted) and multiphonon (dasheddouble-dotted) contributions. Shown in the inset is the vibrational density of states of $\mathrm{H}$ in $\mathrm{MgH}_{2}$ computed from the corrected $12-\mathrm{K}$ spectrum. Bottom: Raman spectra of $\mathrm{MgH}_{2}$ at room temperature (a), and $77 \mathrm{~K}$ (b), obtained using the 488-nm line of the $\mathrm{Ar}^{+}$laser. The low-energy region obtained at $77 \mathrm{~K}$ with the $514.5-\mathrm{nm}$ laser line is also displayed (c). The main peaks are labeled by their assigned symmetry.

Multiple scattering corrections to INS spectra were performed employing a Monte Carlo code devised for the present experimental configuration, based on the procedure described in Ref. 8. In Fig. 1 (top), we show the multiple scattering contribution calculated for $12 \mathrm{~K}$.

In the energy range covered by the experiment, the neutrons are scattered mainly by hydrogen atoms. To extract the vibrational density of states from the experimental data, we have subtracted the contributions from the multiphonon neutron scattering to the measured spectra. These contributions were calculated in an isotropic harmonic approximation using an iterative technique, following the procedure described in Ref. 9. We must emphasize that in the present case, the analysis is based on two assumptions: (a) the vibrational spectrum of the system extends up to $170 \mathrm{meV}$, and (b) for energies below $20 \mathrm{meV}$, the dynamics are described by a Debye spectrum. As a result of the procedure, a refined value of $\left\langle u^{2}\right\rangle$ is obtained. Figure 1 (top) shows the recorded neutron spectra for different temperatures. As seen from the plot, the spectra do not present strong changes in the temperature range covered by the experiment. There are no noticeable frequency shifts, and the differences observed for the studied temperatures can be ascribed to variations in the multiphonon contribution, Debye-Waller factor, and occupation number.

The vibrational spectrum of $\mathrm{MgH}_{2}$ presents several differences with the spectra observed in transition metals and rareearth hydrides, which display a strong H-related optic band separated by a large gap from a very small metal vibration band, ${ }^{10}$ and shares some of the features displayed by $\mathrm{AlH}_{3} .{ }^{11}$
In the present case, the spectrum can be divided into three broad ranges, separated by two gaps between $40-55 \mathrm{meV}$ and $105-115 \mathrm{meV}$. The ranges above $55 \mathrm{meV}$ clearly correspond to H-related optic vibrations and the large widths suggest a large dispersion on the optic phonon branches and, consequently, strong $\mathrm{H}-\mathrm{H}$ interactions. The observed widths could be expected by extension of the features depicted by the dispersion curves and the vibrational density of states of other systems having the rutile structure $\left[\mathrm{MgF}_{2}, \mathrm{MnF}_{2}\right.$ (Ref. 12)]. The optical range of $\mathrm{MgH}_{2}$, in addition, agrees with the low-resolution inelastic spectra of $\mathrm{CaH}_{2}, \mathrm{SrH}_{2}$, and $\mathrm{BaH}_{2}$ reported over a reduced energy range. ${ }^{5}$ The band between 20 and $40 \mathrm{meV}$ can be associated with in-phase vibrations of hydrogen and $\mathrm{Mg}$ atoms. The relatively high intensity of this part of the spectrum indicates that the hydrogen components of the eigenvectors are large for these modes, and the "metal" phonons should be strongly mixed with hydrogen vibrations. 13

The energy range covered by the experiment was chosen according to the results reported from neutron-transmission ${ }^{15}$ and infrared-absorption ${ }^{14}$ experiments, which indicate that the vibrational spectrum extends up to $170 \mathrm{meV}$. We also note that the maximum frequency at which phonon-related infrared absorptions occur (145 meV) (Ref. 1) is very close to the frequency of the highest band observed in the present experiment $(148 \mathrm{meV})$. These results justify hypothesis (a) adopted above for the evaluation of the multiphonon components to the experimental data. On the other hand, assumption (b) was adopted as a reasonable guess for the lowfrequency part of the spectrum, considering that $18 \mathrm{meV}$ was the lower-energy exchange available in the present experiment.

In the analysis that follows, we will consider the $12-\mathrm{K}$ spectrum in order to minimize the multiphonon contribution. The obtained frequency spectrum $Z(\omega)$ is shown in the inset of Fig. 1, evaluated using the procedure previously described and correcting for background, multiple scattering, and multiphonon contributions. In order to test this vibrational density of states, we have computed two derived magnitudes, the incoherent total cross section and the mean-square displacement of hydrogen atoms, and compared them with the available experimental data.

The comparison between the calculated values and the experimental neutron total-cross-section data from Ref. 15 exhibits a very good overall agreement, as can be seen by the plots depicted in Fig. 2(a). In the process of obtaining the calculated DOS, an estimation of $\left\langle u^{2}\right\rangle$ is derived from the multiphonon contributions. The resulting mean-square displacement thus obtained at $295 \mathrm{~K}$ is $\left\langle u^{2}\right\rangle=0.022 \AA^{2}$, which is close to the value $(0.025 \pm 0.002) \AA^{2}$ previously reported. ${ }^{15}$ By applying the described procedure to all the recorded spectra, we have derived the mean-square displacement as a function of temperature displayed in Fig. 2(b). We have also included in this figure for comparison, the $\left\langle u^{2}\right\rangle$ values evaluated from the vibrational density of states shown in Fig. 1. The agreement provides a complementary consistency check of the performed corrections.

We also evaluated the reduced form of the density-ofstates frequency moments using the $12-\mathrm{K}$ neutron-scattering 

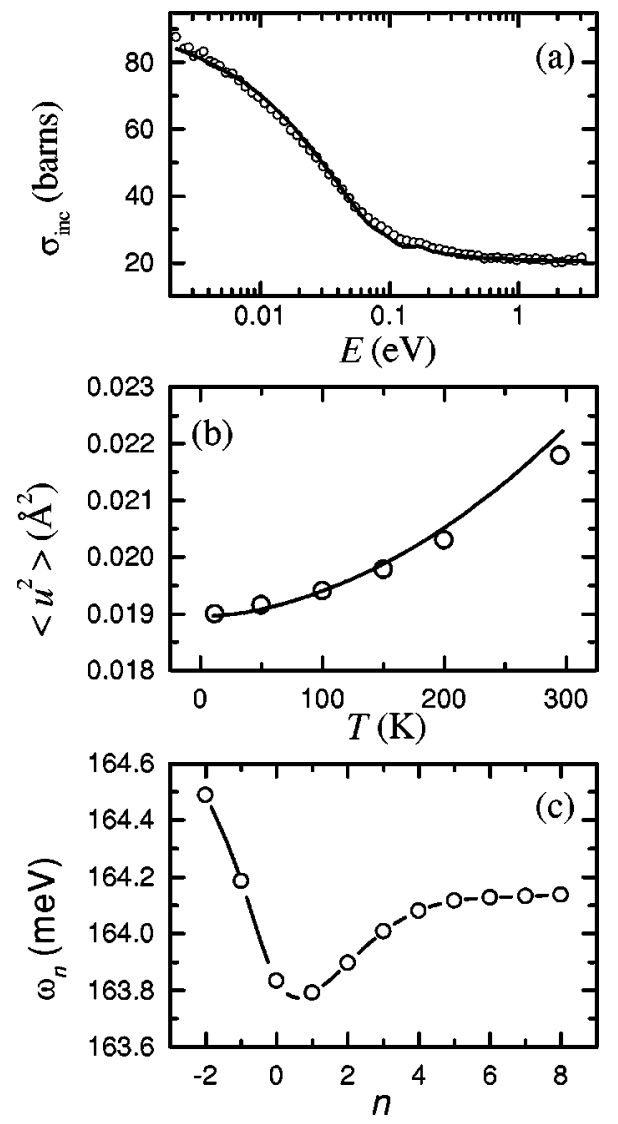

FIG. 2. (a) Comparison between the hydrogen total cross section evaluated from the vibrational density of states (symbols) and the experimental values (line) (Ref. 15). (b) Comparison between mean-square displacement of the proton in magnesium hydride evaluated from the vibrational density of states (line) and values resulting from the analysis of the recorded spectra at each temperature (symbols). (c) Reduced frequency moments for the frequency spectrum corresponding to $12 \mathrm{~K}$ (symbols). The solid line is just a guide to the eye.

data. ${ }^{16}$ These reduced moments, shown in Fig. 2(c), are a measure of the validity of a (Debye) continuum description, for which all should be equal to the Debye frequency. The calculated moments are in fact practically the same, the maximum difference between two of them being less than $0.5 \%$. We note that the $n$ dependence of the moments agrees with the behavior previously observed in alkaline-earth fluorides. ${ }^{17}$ This result shows that integral magnitudes of the frequency spectrum behave as if the density of states were a Debye spectrum with a Debye frequency $\omega_{\mathrm{D}} \approx 164 \mathrm{meV}$, supporting the Debye frequency spectrum assumed in Ref. 1 to describe the vibrational properties of magnesium hydride within $a b$ initio calculations.

We turn now to the Raman spectra, which were recorded in a backscattering configuration, using a Jobin Yvon T64000 equipped with a multichannel detection. The 488-nm and 514.5-nm lines of an $\mathrm{Ar}^{+}$laser were used as the exciting source. Typical resolutions were around $0.15 \mathrm{meV}$. Spectra were acquired at 77 and $300 \mathrm{~K}$, focused at different spots on the sample surface to check for homogeneity. Even for the best studied samples, a background was superimposed to the Raman features. Such a background is probably due to elec- tronic scattering at the magnesium metallic phase, unavoidably mixed with $\mathrm{MgH}_{2}$. To highlight some subtle Raman features intrinsic to $\mathrm{MgH}_{2}$, it has been substracted from the data presented here.

First-order Raman spectra provide the frequencies of zone-center phonons, together with symmetry information important for the identification of bands in the vibrational spectrum. ${ }^{18}$ In addition, second-order processes are sometimes observable that can be related to the phonon density of states. The phonon frequencies and symmetries in the metal fluorides, which have the rutile structure and are thus isomorphous with $\mathrm{MgH}_{2}$, have been originally studied by Porto et al. $^{3}$ There are four Raman-active modes with symmetries $A_{1 g}, B_{1 g}, B_{2 g}$, and $E_{g}$. For all the studied fluorides, these vibrations appear with increasing frequency in the sequence $B_{1 g}, E_{g}, A_{1 g}$, and $B_{2 g}$. The $B_{2 g}$ mode is the weakest, almost unobservable in the spectra, while the $E_{g}$ and $A_{1 g}$ phonons display the strongest scattering efficiency. We are not aware of any such experiment or calculation for the case of $\mathrm{MgH}_{2}$, with the exception of the paper of $\mathrm{Yu}$ and $\mathrm{Lam}^{1}$ where the phonon frequency of the totally symmetric $A_{1 g}$ optical mode is calculated with a frozen-phonon method.

In Fig. 1 (bottom), we present room-temperature and 77-K Raman spectra of $\mathrm{MgH}_{2}$ taken with the 488-nm line of the $\mathrm{Ar}^{+}$laser. The low-energy region obtained with the 514.5-nm laser line is also displayed. The three curves are shown to highlight different features of the spectra: (i) Three main peaks are observed at 37.2, 117.8, and $158.2 \mathrm{meV}$. In all cases, the $117.8-\mathrm{meV}$ peak is the most prominent one. The large background superimposed to the phonon spectra hampered the observation of the higher energy mode at room temperature. (ii) Besides the larger modes, weaker broad bands characteristic of second-order processes can be observed in the energy regions $60-100 \mathrm{meV}$ and $130-190$ $\mathrm{meV}$. And (iii), several small peaks appear at low temperatures below $35 \mathrm{meV}$. These peaks are observed with both laser lines, demonstrating their Raman origin. In addition, their small widths suggest first-order processes.

The assignment of the three main peaks [point (i) above] can be done based on the theoretical calculations of $\mathrm{Yu}$ and $\mathrm{Lam}^{1}$ and from a comparison with the Raman spectra of the isomorphus rutile structure fluoride $\mathrm{MgF}_{2} \cdot{ }^{3}$ In fact, the peak at $158.2 \mathrm{meV}$ coincides almost exactly with the value calculated for the $A_{1 g}$ mode in Ref. 1 (158 meV). On the other hand, the $B_{1 g}, E_{g}$, and $A_{1 g}$ symmetry vibrations in the isomorphous compound $\mathrm{MgF}_{2}$ are, respectively, observed at $11.4,36.6$, and $50.8 \mathrm{meV}$. We can derive an estimation of the corresponding energies in $\mathrm{MgH}_{2}$ considering that they should verify the same frequency ratios in both compounds. This amounts to assuming that the frequencies of these vibrations are simply proportional to some generalized force constants, and to the inverse of the mass square root. Under this assumption, the $B_{1 g}$ and $E_{g}$ vibrations in $\mathrm{MgH}_{2}$ are expected to appear, respectively, at 35.5 and $113.6 \mathrm{meV}$, which agree within $4 \%$ with the measured values.

The spectral position of the three observed Ramanallowed modes coincides with the boundaries of some of the larger structures in the vibrational density of states derived from the INS data, as expected for zone-center excitations. 
Thus, their assignment sets the symmetry of some of the phonon bands contributing to this spectrum. On the other hand, the energy of the second-order smaller features [point (ii) above] corresponds to $\omega$ and $2 \omega$ of one of the larger bands in the INS data, which does not coincide with any Raman peak. We conclude that the latter must be infrared active in a first-order process, and thus become Raman active in second order (two-phonon scattering) explaining the appearance of scattering at $2 \omega(130-190 \mathrm{meV}){ }^{18}$ The weak band at $\omega$, however, cannot be understood from symmetry and kinematic grounds and, thus, is probably due to disorderinduced effects. Such effects as, e.g., "impurity scattering," might activate the otherwise Raman-forbidden vibrations. The presence of disorder cannot be excluded in these $\mathrm{MgH}_{2}$ samples: due to the preparation method, proton vacancies are highly probable because of partial filling of the hydrogen sites. The existence of clusters with empty hydrogen sites (and, thus, reduced number of bonds) might also explain the origin of the series of weak Raman peaks observed in the low-energy region. However, supercell or shell-model calculations of structures with variable hydrogen content are needed to establish this point.
In summary, we have presented INS results on magnesium hydride. The strong incoherent scattering by hydrogen atoms enabled us to extract the $\mathrm{H}$ vibrational density of states and to compute several derived magnitudes. These results reveal that the dynamics of the proton in $\mathrm{MgH}_{2}$ cannot be described in terms of localized vibrations within a singleparticle potential, as it has already been applied to most groups-III-VII metal hydrides. Moreover, for computing integral magnitudes, the density of states can be aproximated by a Debye spectra with $\omega_{\mathrm{D}}=164 \mathrm{meV}$. Three main peaks are observed in the Raman spectra and have been assigned to the $A_{1 g}, B_{1 g}$, and $E_{g}$ symmetry Raman-allowed vibrations. Several other smaller features appearing in the spectra are probably due to two-phonon and disorder-induced processes. The Raman spectra show good agreement with the INS data, thus contributing to the identification of the strongest bands in the derived phonon density of states of $\mathrm{MgH}_{2}$.

We wish to thank J.R. Granada and J. Sofo for helpful discussions, J.L. Capararo for the sample preparation, and P. Palleau for his assistance during the experiment at ILL. This work was partially supported by ANPCYT (Argentina) through Grant No. PICT No. 03-00000-00680.
*Also at CONICET, Argentina.

${ }^{1}$ R. Yu and P.K. Lam, Phys. Rev. B 37, 8730 (1988).

${ }^{2}$ The lattice dynamics of these fluorides has been discussed in R.S. Katiyar, J. Phys. C 3, 1693 (1970) $\left(\mathrm{MgF}_{2}\right)$; J.P. Hurrel and V.J. Minkiewicz, Solid State Commun. 8, 463 (1970) $\left(\mathrm{BaF}_{2}\right)$; and W.J. Buyers, Phys. Rev. 153, 923 (1967) (NaF).

${ }^{3}$ S.P.S. Porto, P.A. Fleury, and T.C. Damen, Phys. Rev. 154, 522 (1967).

${ }^{4}$ R. Almairac, J.L. Sauvajol, C. Benoit, and A.M. Bon, J. Phys. C 11, 3157 (1978); R. Almirac and C. Benoit, ibid. 7, 2614 (1974).

${ }^{5}$ A.J. Maeland, J. Chem. Phys. 52, 3952 (1970).

${ }^{6}$ W.H. Zachariasen, C.E. Holley, and J.F. Stampfer, Acta Crystallogr. 16, 352 (1963).

${ }^{7}$ J.R. Santisteban, J.R Granada, and R.E Mayer, J. Neutron Res. 7, 1 (1998).

${ }^{8}$ J. Dawidowski, F.J. Bermejo, and J.R. Granada, Phys. Rev. B 58, 706 (1998).

${ }^{9}$ D.K. Ross, V.E. Antonov, E.L. Bokhenkov, A.I. Kolesnikov, E.G. Ponyatovsky, and J. Tomkinson, Phys. Rev. B 58, 2591 (1998); A.I. Kolesnikov et al., Physica B 174, 257 (1991).
${ }^{10}$ Complete reviews of the dynamics of hydrogen in transitionmetal and rare-earth hydrides can be found in T. Springer, $H y$ drogen in Metals (Springer-Verlag, Berlin, 1978), Vols. I and II; Y. Fukai, The Metal-Hydrogen System (Springer-Verlag, Berlin, 1993); and A. Furrer, Neutron Scattering from Hydrogen in Materials (World Scientific, Singapore, 1994).

${ }^{11}$ A.I. Kolesnikov, M. Adams, V.E. Antonov, N.A. Chirin, E. Goremychking, Y. Markushkin, M. Prager, and I.L. Sashin, J. Phys.: Condens. Matter 8, 2529 (1996).

${ }^{12}$ M. Mostoller and J.C. Wang, Phys. Rev. B 32, 6773 (1985).

${ }^{13}$ B. Dorner, E.L. Bokhenkov, S.L. Chaplot, J. Kalus, J. Natkaniec, G.S. Pawley, U. Schmezer, and E.F. Sheka, J. Phys. C 15, 2353 (1982).

${ }^{14}$ C. Stander and R. Pacey, J. Phys. Chem. Solids 39, 829 (1978).

${ }^{15}$ J.R. Santisteban, J.R. Granada, and J. Dawidowski, Physica B 269, 345 (1999).

${ }^{16}$ T.H.K. Barron et al., Adv. Phys. 29, 609 (1980).

${ }^{17}$ B. Yates, Thermal Expansion (Plenum Press, New York, 1972).

${ }^{18}$ M. Cardona, in Light Scattering in Solids, edited by M. Cardona and G. Güntherodt (Springer, Berlin, 1982), Vol. 2. 